(system S), and $l_{0}$ their distance in the natural state (system $\mathrm{S}_{0}$ ), these distances being both determined in the manner specified in what precedes. Then

$$
\xi \xi=\left(l-l_{0}\right) l_{l_{0}} .
$$

Again, if $\mathrm{P}^{\prime \prime}$ is the particle $\xi, \eta+d \eta, \xi$, and if the angle $\mathrm{P}^{\prime} \mathrm{PP} /$, calculated as stated before, has in the two cases the values $\delta$ and $\delta_{0}\left(=\frac{1}{2} \pi\right)$, we shall have

$$
\xi_{\eta}=\delta_{0}-\delta
$$

The six deformations $\xi_{\xi}$. . . will be considered as infinitely small. In the problem we have in view, they are of the order of magnitude $v^{2} / c^{2}$, so that our final result will be correct to that order.

If we put

$$
\begin{array}{r}
\mathrm{U}=\mathrm{A}\left(\xi \xi^{2}+\eta_{\eta}{ }^{2}+\zeta \xi^{2}\right)+\frac{1}{2} \mathrm{~B}\left(\xi_{\xi}+\eta_{\eta}+\zeta \xi^{2}+\right. \\
\frac{1}{2} \mathrm{~A}\left(\xi_{\eta}^{2}+\eta \zeta^{2}+\zeta \xi^{2}\right),
\end{array}
$$

the well-known expression for the potential energy of an isotropic elastic body, $U$ will be invariant for any change of co-ordinates.

As to the kinetic energy $\mathrm{T}$, it is to be replaced by an expression containing $\rho \frac{d s}{d t}$. Finally, we must write, instead of (3),

$$
\delta \int_{t_{1}}^{t_{2}} \int\left(-c \rho-\frac{\mathrm{I}}{c} \mathrm{U}\right) \frac{d s}{d t} d t d \xi d \eta d \zeta=0
$$

We have still to add the formulæ that are found by working out the above definitions of $\xi_{\xi}, \xi_{\eta}$, etc., viz. :--

$$
\begin{gathered}
\xi_{\xi}=-\frac{1}{2} \Sigma(a \beta) g_{a \beta} \frac{\partial x_{a}}{\partial \xi} \frac{\partial x_{\beta}}{\partial \xi}+\frac{\left[\Sigma(a \beta) g_{a \beta} v_{\beta} \frac{\partial x_{a}}{\partial \xi}\right]^{2}}{\Sigma \Sigma(a b) g_{a b} v a v_{b}}-\frac{1}{2}, \\
\xi_{\eta}=-\Sigma(\alpha \beta) g_{a \beta} \partial x_{a} \frac{\partial x_{\beta}}{\partial \xi}+\frac{\Sigma(a \beta) g_{a \beta} v_{\beta} \frac{\partial x_{a}}{\partial \xi} \cdot \Sigma(a \beta) g_{a \beta} v_{\beta} \frac{\partial x_{a}}{\partial \eta}}{\Sigma(a b) g_{a b} v a v_{b}}
\end{gathered}
$$

$\left\langle v_{1}, v_{2}, v_{3}\right.$ are the components of the velocity, and $v_{4}=\mathrm{I}$ ).

In our problem the body is supposed to move in a normal system of co-ordinates. By this our formulæ simplify to ${ }^{1}$

$$
\begin{gathered}
\delta \int_{t_{1}}^{t_{2}} \int\left(-c^{2} \rho-U\right)\left(1-\frac{v^{2}}{c^{2}}\right)^{\frac{1}{2}} d t d \xi d \eta d \zeta=0, \\
\xi_{\xi}=\frac{1}{2} \Sigma(a)\left(\frac{\partial x_{a}}{\partial \xi}\right)^{2}+\frac{\left[\Sigma(a) v_{a} \frac{\partial x_{a}}{\partial \xi}\right]^{2}}{2\left(c^{2}-v^{2}\right)}-\frac{1}{2},
\end{gathered}
$$

1 If in (7) we replace $\left(r-z^{2} / c^{2}\right)^{\frac{1}{4}}$ by $x-z^{2} / 2 c^{2}$, omitting the constant term $-c^{2} \rho$ and neglecting $U . z^{2} / 2 c^{2}$, we are led back to the ordinary formula (3).

$$
\xi_{\eta}=\Sigma(a) \frac{\partial x_{a}}{\partial \xi} \frac{\partial x_{a}}{\partial \eta}+\frac{\Sigma(a) v_{a} \frac{\partial x_{a}}{\partial \xi} \cdot \Sigma(a) v_{a} \frac{\partial x_{a}}{\partial \eta}}{c^{2}-v^{2}}
$$

When applied to a revolving body, these equations will enable us to determine the deformation that is produced, wholly independently of the theory of relativity, by centrifugal force, a deformation that will in reality far surpass the changes we want to consider. To get free from it we can consider the ideal case of a "rigid" body-i.e. a body for which the moduli of elasticity $A$ and $B$ in (6) are infinitely great. The centrifugal force will then have no effect on the dimensions, but the changes required by the theory of relativity will subsist. The assumption has also the advantage of simplifying the calculations; indeed, since $U$ becomes infinitely great, the term $-c^{2} \rho$ in (7) may be omitted.

I have worked out the case of a thin circular disc rotating with constant speed about an axis passing through its centre, at right angles to its plane. The result is that, if $v$ is the velocity at the rim, the radius will be shortened in the ratio of $\mathbf{x}$ to $\mathbf{I}-\frac{\mathbf{I}}{8} \frac{v^{2}}{c^{2}}$. The circumference changing to the same extent, its decrease is seen to be exactly one-fourth of that of a rod moving with the same velocity in the direction of its length. That there would be a smaller contraction was to be expected; indeed, the case can be compared to what takes place when a hot metal band is fitted tightly around a wheel and then left to cool.

At first sight our problem seems to lead to a paradox. Let there be two equal discs $A$ and $B$, mounted on the same axis, A revolving and B at rest. Then $\mathrm{A}$ will be smaller than $\mathrm{B}$, and it must certainly appear so (the discs. being supposed to be quite near each other) to any observer, whatever be the system of co-ordinates he chooses to use. However, we can introduce a system of co-ordinates $\mathrm{S}^{\prime}$ revolving with the disc $\mathrm{A}$; with respect to these it will be $B$ that rotates, and so one might think that now this latter disc would be the smaller of the two. The conclusion would be wrong because the system $S^{\prime}$ would not be a normal one. If we leave $\mathrm{S}$ for it, we must at the same time change the potentials $g_{a b}$, and if this is done the fundamental equation will certainly again lead to the result that $A$ is smaller than $B$.

\title{
The Geometrisation of Physics, and its Supposed Basis on the Michelson-Morley Experiment.
}

\section{By Sir Oliver Lodge, F.R.S.}

SO much has been written about the Michelson. $\checkmark$ Morley experiment that it would be needless to refer to it here, had it not been interpreted by philosophic writers in an interesting but overviolent ard, as some think, illegitimate manner. Historically it really does lie at the root of the remarkable attempt which is being made to geo- metrise physics, and to reduce sensible things like weight and inertia to a modification of space and time. The work of great Geometers has been pressed into the service, and a differentialinvariant scheme of expression has been utilised to do for physics in general, and especially for gravitation, what Maxwell's equations did for No. 2677 , VOL. IO6] 
electric and magnetic forces. The prominent merit of these equations is that they replace any apparent predilection for forces acting at a distance, by explicit recognition of a modified medium (or at least a modified space) in contact with the accelerated particle.

The beauty and ingenuity of this scheme, and of the reasoning associated with it, are apt to overpower the judgment at times, and to lead to pseudo-philosophic conclusions which are not really justified, and some of which are repugnant to common-sense. It is scarcely wise to seek to interpret physically every link in a chain of mathematical reasoning. If the chain is coherent and if the terminal hooks are firm-that is, if the end results are intelligible and verifiable-no more need be expected from any system of equations. The astonishing thing is that statements of such extensive generality can be written in so compact a form, and that so considerable a range of experience can be summarised in terms of a pure hypergeometry, even though that be of a complex character.

It is undeniable that mathematicians, with a self-denying ordinance about coefficients, can thus attain remarkable criteria, and are able to anticipate definite results; but we need not seek to engraft their modes of expression on the real world of physics. We need not consider realities superseded, because a system of pure space and time can be devised which can formulate, and be consistent with, the movements observed by ordinary men and animals. What we observe is not motion and position alone : a wealth of colour, form, and beauty is also within our ken, and will not readily evaporate into a geometrical modification of empty space.

The Relativity argument is based on a policy of exclusion. It rejects everything that seems unnecessary; it dispenses with many of our longstanding conceptions; and accordingly is hailed as a simplification. The first simplification was the denial of any test for motion through a continuous fundamental medium, and a consequent ignoring of such a medium. The second step was to eliminate gravitational and other forces, with further denial of a power of discrimination between different kinds of acceleration. A third simplification, and further introduction of coefficients, enabled electro-magnetic forces to be similarly eluded. And if our conceptions permit of any further simplification, perhaps the additional properties of matter studied by Chemists and Biologists and Artists may be extruded too, and the rich fullness of the universe be impoverished into a mental abstraction.

To summarise, then :-

In such a system there is no reed for Reality; only Phenomena can be observed or verified : absolute fact is inaccessible. We nave no criterion for truth; all appearances are equally valid; physical explanations are neither forthcoming noi required; there need be no electrical or any other theory of the constitution of matter. Matter is, indeed, a mentally constructed illusion generated No. 2677 , VOL. IO6] by local peculiarities of Space. It is unnecessary to contemplate a continuous medium as a universal connector, nor need we try to think of it as suffering modification transmitted from point to point from the neighbourhood of every particle of gravitational or electrified matter : a cold abstraction like a space-time-manifold will do all that is wanted, or at least all that the equations compel. And, as a minor detail, which will bring us to the point, it is not necessary to invoke a real FitzGerald contraction in order to explain the result of the Michelson Experiment.

\section{The Experiment.}

With this prelude, which merely summarises a great deal more that in time I wish to say, let us proceed to the M.M. Experiment and recall attention to what it really does demonstrate rather than to what it is too often imagined to prove.

Take a horizontal square slab of stone (or wood), float it above an annular trough of mercury so that it can be steadily turned round a vertical axis. Fix mirrors to its surface, so arranged that a beam of light, split into halves, can be sent, one half to and fro lengthways, and the other half to and fro breadthways, each half travelling an equal distance as fixed by the slab. Reunite the two halfbeams, observe the interference bands so formed, and see if they shift in a periodic manner during a leisurely rotation of the stone and observer through all azimuths.

If light is really a wave and not a projectile motion, and if we are living in a virtual drift of æther due to our real orbital motion through that medium, and if the stone or other material of the slab preserves and determines unalterably the actual distances travelled by the two half-beams of light in the interval between their split and their reunion-then the bands should undergo a sinuous shift, with amplitude representing a lag of the order $1^{-8}$, as the stone revolves.

But, on the electrical theory of matter, cohesion is a residual chemical affinity or electrostatic attraction; and such attraction is known to be modified electrokinetically if the charged atoms are in rapid motion through the dielectric medium; hence it becomes not only possible but likely that the dimensions of the slab will change if we are moving through the æther; the square being subject to a slight distortion. And the change metrically to be expected, in accordance with electrical theory, is found on examination to be also of the order $\mathrm{IO}^{-8}$, and in fact was ultimately shown by Larmor to be of a precisely compensating character. (" Ether and Matter," I900, p. I75.)

The fact is that the lines of force on an isolated charged sphere redistribute themselves, if it be moving through the æther, as if it had become a stationary oblate spheroid with axis in the direction of motion. Such a spheroid becomes the static representation of a moving sphere, and may be held to take its place as the most symmetrical figure; for the only way in which we can infer the 
virtual shape of an electron is by the distribution of its field.

Ether being incompressible, I expect that the change is really a pure constant-volume distortion, consisting of a pair of lateral extensions and a longitudinal contraction, as suggested in my B.A. address "Continuity," I9I 3 , in the Birmingham volume, p. 25 (or in a separate publication by Dent and Son, pp. $5^{8}$ and III). But all that is significant for present purposes is the ratio of the longitudinal to the lateral change-a ratio commonly spoken of as the FitzGerald or FitzGerald-Lorentz contraction.

It is customary to consider this as only a longitudinal shrinkage, but it is just as easy to allow for a possible lateral change too :-

The velocity of light through the medium being always $c$, and the speed of the light-conveying medium relatively to the matter of the block being $v$, the effective or resultant speed for the to-andfro cross-current light-journey is $\sqrt{ }\left(c^{2}-v^{2}\right)$, while for the up- and down-stream journeys the effective speeds are $(c-v)$ and $(c+v)$ respectively.

The intended perpendicular distances marked out on the slab being both $x$, the distance alongstream becomes, say, $\beta x$ (whichever side of the slab gets periodically into that position as it rotates), and the distance across-stream may be called $\gamma x$; so the respective single journeys are

$$
\begin{array}{ll}
\text { (along) } & \beta x=(c-v) t_{1}=(c+v) t_{2} \\
\text { (across) } & \gamma x=\sqrt{ }\left(c^{2}-v^{2}\right) t_{3} .
\end{array}
$$

The observation does not consist in measuring either $t_{1}$ or $t_{2}$ or $t_{3}$; nor is even $x$ measured with precision. The whole object of the experimenter is to measure the small excess,

$$
t_{1}+t_{2}-2 t_{3}
$$

to express it as a fraction of wave length, and to compare it with the distance $2 x$. Repeated observations show that the excess is actually zero. And with this additional datum,

$$
t_{1}+t_{2}=2 t_{3} \text {, }
$$

it is mere algebra to reckon that the necessary effective contraction is

$$
\beta / \gamma=\sqrt{ }\left(\mathrm{I}-v^{2} / c^{2}\right) ;
$$

while if we choose to add the constant volume relation, $\beta \gamma^{2}=\mathrm{I}$, we get also

$$
\gamma=\left(\mathbf{I}-v^{2} / c^{2}\right)^{-\frac{1}{6}} .
$$

The experiment therefore verifies the FitzGerald contraction, and tends to confirm the electrical theory of matter.

I was interested, when visiting the University of Chicago last winter, to find that Prof. Michelson himself was perfectly satisfied with this sort of view of his experiment, and did not consider that its interpretation necessitated any revolutionary considerations. The FitzGerald contraction is a peculiarity which could scarcely have been detected in any other way, since it is really an affair of the æther-the connecting medium in which all molecules are embedded-and affects every kind of matter to the same extent.

$$
\text { No. } 2677 \text {, VOL. IO6] }
$$

An objection has been raised, with apparent seriousness, that this contraction cannot be real, since, if it were, the rim of a spinning-wheel would contract more than the spokes, and so the ratio of circumference to diameter would not be $\pi$. This is an instructive and rather humorous example of the prevalent tendency to control physics by geometry. We might argue similarly that the rim of a wheel could not be a fraction of a degree cooler than the spokes; and it might be heldindeed it has been held-that the state of strain in an actual rotating wheel or disc would require non-Euclidean geometry to express it. If the propositions of geometry require physical measurements to sustain them, they can scarcely be of the kind we have been accustomed to associate with the name of Euclid. And if a special geometry has to be invented in order to account for a falling apple, even Newton might be appalled at the complications which would ensue when really difficult problems are tackled. Nevertheless, that is the kind of geometry to which relativity introduces us-a geometry based on hypothetical laboratory measurements with scales and clocks, and one the propositions of which can be interfered with by metrical observations. It is therefore sometimes called a "natural" geometry, free from metaphysics; it might equally well be called an abstract sort of theoretical physics, and not geometry at all.

\section{Relativity Explanation of the Experiment.}

It is well known that the simple interpretation above given of the M.M. experiment is not palatable to relativists; they consider that it is a forced and arbitrary explanation, and that they can account for the M.M. result more naturally by employing a geometrical device and by applying certain general hypotheses. The Principle of simple relativity is that a transformation to uniformly moving axes can make no difference to anything essential; and the accompanying obsession is that no observer can detect any apparent change in the velocity of light.

In order to apply these principles, the method adopted by a Relativist is to take two observers instead of one, to supply them with personal clocks and measuring rods, and then to make one of them fly through the laboratory at speed $v$; thus rendering accurate measurement rather difficult for him, and introducing some confusion into his ideas of space and time - especially as he is not to be allowed to know that he is moving. He may be at rest in the æther, but everything not attached to him or to his medium will be rushing along; accordingly objects will appear to be contracted, and all clocks but his own will seem to go slow. ${ }^{1}$ The only thing

1 It is not easy to explain without symbols why earthbound clorks should appear to go slow to an aviator, and an aviator's clock appear $t$, go slow to a man on the ground. The plain man wonld think that they would both appear to go fast during approach, and slow during recession; hut the meaning is not so simple as that. Nor is it because a pendulum bas
lengthened, or anything phys'cal or real of that sort. The argument lengthened, or anything phys'cal or real of that sort. The argument
appears to be that the other man's clock must be estimated as relatively appears to be that the other man's clock must be estimated as relatively
slow, by each of two observers moving relatively to each other, because slow, by each of two observers moving relatively to each other, because otherwise they could measure different velocities of light; which, though
not repugnant to common-sense, is contrary to the basic Principle of not repugnant
Relativity. 
unaltered is speed, for he is allowed to compensate every confusion about space by an equal opposite confusion about time. However, he is provided with an instantaneous camera for taking snapshots which he can afterwards. study, and he does his best; he makes his observations of times and distances, and records them with dashed letters. Meanwhile the other more comfortably situated observer, attached to the slab and the laboratory furniture (who also considers himself at rest, though the first man may think of him as rushing through the ather), records, undashed, the readings of his peculiar instruments too.

A relativist, not caring in the least which or whether either of these sets of measurements has any absolute meaning, but assuming them to be made accurately, applies the transformation of Lorentz and Larmor for change of co-ordinates between sets of axes moving relatively to each other; and thereby finds that if the laboratory readings are $x$ and $t$, while the flying man's readings are $x^{\prime}$ and $t^{\prime}$ (each being supposed to have a local space and time of his own, absolute space and time having no meaning), the following relations must hold-

$$
x^{\prime}=\frac{x-v t}{\sqrt{\left(1-v^{2} / c^{2}\right)}} ; \quad t^{\prime}=\frac{t-v x / c^{2}}{\sqrt{ }\left(\mathrm{I}-v^{2} / c^{2}\right)}
$$

From these simple but important, and indeed fundamental, equations it comes out that if $x^{\prime} / t^{\prime}=c$ for the flying man, then $x / t=c$ for the other observer likewise. So the result is just as if nothing at all was moving, except light, and in fact as if bodily motion through the light-conveying medium were meaningless. Hence everybody should be satisfied, without any appeal to, or confirmation of, any physical theory whatever. The null (or dull) result of the experiment now requires no explanation; there is no need even to emphasise the double to-and-fro journey of the light; single journeys serve; for the apparent speed of light is (really, has been assumed) the same in all directions, no matter what it be referred to. Distances and times may appear different to different observers, but they are arbitrary or conventional appearances at best, and they tend to compensate each other.

Hence arises the $r=c t$ and $r^{\prime}=c t^{\prime}$ superstition, about the concentricity of a wave front round each one of a group of observers initially at the origin however much they may have scattered since; because, apart from gravitation, $x^{2}+y^{2}+z^{2}-c^{2} t^{2}$ must be independent of axes of reference; or, what is the same thing in our simple case,

$$
\frac{x-c t}{x^{\prime}-c t^{\prime}}=\frac{x^{\prime}+c t_{1}^{\prime}}{x+c t^{\prime}}=\text { constant }=\sqrt{\frac{c-v}{c+v}} \text {. }
$$

And the other peculiarities of the simpler theory of relativity immediately follow: some of them very surprising and interesting.

For instance, take the composition of motions :

If a thing is moving with speed $u=x^{\prime} / t^{\prime}$, relatively to an origin which itself is moving in (say) the same direction with speed $v$ referred to a fixed origin, then the resultant speed $w=x / t$, referred to the same fixed origin, will be obtained by aid of the above transformation for moving axes reduced to relative rest--

$$
x=\beta(x+v t) ; t=\beta\left(\iota^{\prime}+v x^{\prime} / c^{2}\right) ;
$$

whence we get

$$
w=\frac{x}{t}=\frac{u+v}{\mathrm{I}+u v / c^{2}}
$$

a curious expression for the combined velocities. [Likening it to a rotation of axes, with $v / c=\tanh \theta$, the summation is not $\tanh \theta_{1}+\tanh \theta_{2}$ but $\tanh \left(\theta_{1}+\theta_{2}\right)$.] It gives simple summation for slow speeds, and an unattainable maximum $c$ for high speeds. If either or both of the component velocities attain the magnitude $c$, the resultant $w$ attains the same maximum, and cannot exceed it. But the most notable property of this expression is that it gives practically the same expression as Fresnel's æther-intuition gave, for light travelling down a stream of water-an expression verified by Fizeau's famous experiment. For let the speed $u$ be that of light inside a dense material medium, say $c \div \mu$, and let the material medium be itself travelling in the same direction at speed $v$, then the resultant speed of the light would be, by the above expression,

$$
w=\frac{c^{2}+\mu v c}{\mu c+v}
$$

or, what is the same thing,

$$
w=c / \mu+v \cdot \frac{\mathrm{I}-\mathrm{I} / \mu^{2}}{\mathrm{I}+v / \mu c}
$$

And this, to a high degree of approximation, is practically identical with the Fresnel-Fizeau result, viz. :

$$
w=c / \mu+v\left(\mathrm{I}-\mathrm{I} / \mu^{2}\right)
$$

That such a result-which was supposed to give some kind of information about the behaviour of æther inside dense matter-can be obtained irrationally by a simple geometrical device, is surely surprising.

Again, the variation of the mass factor in momentum, which was originally predicted from the electrical theory of matter and afterwards verified, exhibits itself as an outcome of the relativity expression without any physical theory at all. The inertia of an electric charge, and indeed of all energy, seems to come out likewise. Light has a mass-factor, but not a conserved or invariable one, while matter has both a fixed and a variable term in its factor; which is a notable and suggestive pair of facts.

From the point of view of the above relativity composition of velocities, the Michelson-Morley result is obvious; for if the speed $u$ under observation is already $c$, as it is in that experiment, it is useless to compound another velocity, $\perp-v$, with it; because, if that is the law of composition, the resultant velocity still comes out $c$, no more and no less.

It may be said that whereas most experimenters assume an absolute time, the relativist assumes one absolute unattainable or unexceedable velo-

No. 2677 , VOL. IO6] 
city. That is the assumption on which his whole argument is based; and, as an instinctive intuition, there may be a foundation for it (see below), and even for the further assumption that the fundamental absolute velocity is either equal to, or of the same order as, the velocity of light.

It must not be supposed however that the Michelson-Morley experiment substantiates the structure thus begun upon it. It does not really prove that the velocity of light in a moving medium is the same in all directions; that is a gratuitous but fundamental hypothesis, not really based on any experiment-certainly not on one which only deals with to-and-fro journeys over matter-fixed distances. Nevertheless, whether or not this foundation-stone of Relativity is well and truly laid, the structure built over it is of remarkable interest; and possibly can sustain itself by its own consistency, and by its attachments to other facts, even if it be admittedly arched over the particular experiment on which it was originally supposed to be founded.

\section{A Few Remarks on the More General Theory of Relativity.}

The consistency of all these things, including the conservation of energy and momentum, with simple relativity, amply accounts for the enthusiasm with which brilliant mathematicians, untrammelled by a sense of physical reality, have absorbed and developed the idea.

Indeed, as we know, Einstein was enticed to go on and try a transformation to accelerated axes also, to identify gravitation and inertia, to ally both with the so-called centrifugal force, and to reduce them all to a still more elaborate geometry, in which the Lorentz transformation is inadequate, and Euclidean propositions are superseded. $\mathrm{He}$ found ready to his hand a recondite scheme, provided for other and less worldly purposes by Gauss and Riemann; but, to make use of it, he had to introduce a fresh agnostic principle, a Principle of Equivalence. He must assume that we cannot discriminate between a whirling table and a gravitational field; that we have no criterion between a falling apple and a rising Earth; and that it is only prejudice which makes us feel assured about a rotating Earth, and unwilling to contemplate a diurnally revolving star. Geometrically they are all the same; and a suitable space-time system can be devised, and expressed in equations, which will account for, or at least express, most things observed in astronomy, and some additional things more allied with physics.

Now we must admit that if we are permitted to discard relativity as a philosophy and accept it as a method, the form of the most striking of its equations is advantageous, and represents an advance in symbolism, quite apart from any unphysical contributions to its origin and any lack of legitimacy about its birth. For the weight of an apple must be really, as it always has been hypothetically and vaguely, attributable to a gravitationally modified æther pressing down on each particle. Similarly the path of a planet from inNO. 2677 , VOL. IO6] stant to instant can be expressed more intimately by a variational equation, involving always the next step, than by an action-at-a-distance formula, or by a merely kinematic summary which integrates the result over an entire orbit. But this powerful and ingenious method of research can be interpreted, in words, so as to suggest nothing more than a warping of an unreal emptiness-a sort of return to Descartes' vortices without their imagery, a rejection of Copernicus and Galileo and Newton.

Whereas what is really wanted for a truly Natural Philosophy is a supplement to Newtonian Mechanics, expressed in terms of the medium which he suspected and sought after but could not attain, and introducing the additional facts, chiefly electrical-especially the fact of variable inertiadiscovered since his time. Such a philosophy would insist that the specific state of the medium throughout a gravitational field must be the immediate consequence, indeed the absolute essence, of the existence of each material particle; their separate potentials being combined by simple addition without any second-order complications. Equally an electric field must be part of the actual constitution of an electron, and inseparable from it : though the compounding of electric fields need not be so simple a matter, since charges in proximity do interfere with each other.

If we could understand the structure of the particle, in terms of the medium of which it is composed, and if we knew the structure of the rest of the medium also, so as to account for the potential stress at every point--that would be a splendid step, beyond anything accomplished yet. But that the particle is a singularity in the medium, and that its inertia and gravitational field are essential to, and part of, its very existence, must certainly be true; and this is what the Einstein theory, in its own peculiar geometrical unphysical way, has grasped. There are not half a dozen diverse but interlocked things in Nature: there is one definite quiescent medium, full of exceedingly fine-grained turbulent energy, with consequent properties which, when unravelled, will supply the key to all the non-mental phenomena occurring in it. Provided always that the locking up of small regions of this turbulence, not merely in the travelling form of light-waves, but in the potentially stationary form of electrons, can also be explained and understood.

Relativity, as a consecutive point-to-point method of arriving at results, is a first step towards this ideal, but it is not a Newtonian step; it is rather a blindfold method of investigation, like Entropy and Least Action.

\section{The Fundamental Velocity.}

To make a philosophic scheme of existence complete, more than the expression of a static instant is required, there must be duration likewise: the universe is not merely a Being but truly a Becoming; Time has to be associated with Space. This can only be done by a velocity factor of some kind; but to arrive at an appropriate factor, rela- 
tivists have not dived down into the æther, as someone must ultimately dive, and dissected out the intrinsic speed of its turbulence-which is really the fundamental velocity in existence-they have utilised the more obvious and conspicuous consequence of this fundamental speed, viz. the uniform velocity with which the æther can convey a great variety of signals. Indeed their attachment to appliances like clocks and rods has led them to pay almost undue reverence to the æther's power of transmitting waves at a high and otherwise unattainable speed. It is true that these waves are among our methods of receiving and conveying information; but too much attention may be paid to the mere reception of information; and what is spoken of as "warping" is not limited to space alone. For some philosophers speak as if the duration of an event could be extended by merely delaying the reception of the news of its end; as if we could prolong a man's life by evading the tidings of his death, and might be entitled to say, without absurdity, that a man who died at seventy had lived seventy-one years and a lot of miles, if we had travelled so far that the messenger took a year to reach us. That such things can be gravely uttered is surely a tribute to the beauty and complexity of the mathematical scheme which can temporarily so warp the judgment even of the most competent.

If I am wrong in this I share a fraction of rashness with the admirable audacity of Einstein in the Weltmacht oder Niedergang sort of attitude which he takes up about his predicted shift of spectral lines. I feel a doubt whether those lines will be found shifted-at least when the observation is made outside a strong gravitational field-but I should be quite content either way, and would not think of asking anyone to abandon the method of relativity on that account. ${ }^{2}$

For undoubtedly general relativity, not as a philosophic theory but as a powerful and comprehensive method, is a remarkable achievement; and an ordinary physicist is full of admiration for the equations and the criteria, borrowed from hyperGeometers, applied by the genius of Einstein, and expounded in this country with unexampled thoroughness and clearness by Eddington. But, notwithstanding any temptation to idolatry, a physicist is bound in the long run to return to hts right mind; he must cease to be influenced unduly by superficial appearances, impracticable measurements, geometrical devices, and weirdly ingenious modes of expression; and must remember that his real aim and object is absolute truth, however difficult of attainment that may be, that his function is to discover rather than to create, and that beneath and above and around all Appearances there exists a universe of full-bodied, concrete, absolute, Reality.

2 Since the above paragraph was in type a Circular, dated January, 1921, has reached me from Prof. Slipher, of the Lowell Observatory a Flagstaff, Arizona, recording an extravagant rate of recession-something like a thousand miles a second-for two specified nebula, presumably of the spiral class. Rut both nebulx are reported to have large and billiant nuc ei ; and if the concentration of their aggregate mass were sufficientthat is, if their $M / R$ or $\rho^{k_{2}}$ were some two or three thousand's of times greater than that of our sun, and therefore of a totally different order from that of our stellar system-the observation could be interpreted, not as recessic $n$, but as an Einstein shift of speciral lines.

\section{Electricity and Gravitation}

By Prof. H. Weyl.

[Translated by Dr. Robert W. Lawson.]

MODERN physics renders it probable that the D only fundamental forces in Nature are those which have their origin in gravitation and in the electromagnetic field. After the effects proceeding from the electromagnetic field had been co-ordinated by Faraday and Maxwell into laws of striking simplicity and clearness, it became necessary to attempt to explain gravitation also on the basis of electromagnetism, or at least to fit it into its proper place in the scheme of electromagnetic laws, in order to arrive at a unification of ideas. This was actually done by $\mathrm{H}$. A. Lorentz, G. Mie, and others, although the success of their work was not wholly convincing. At the present time, however, in virtue of Einstein's general theory of relativity, we understand in principle the nature of gravitation, and the problem is reversed. It is necessary to regard electromagnetic phenomena, as well as gravitation, as an outcome of the geometry of the universe. I believe that this is possible when we liberate the world-geometry (on which Einstein based his theory) from an inherent inconsistency, which is still associated with it as a consequence of our previous Euclidean conceptions. NO. 2677 , VOL. IO6]
The great accomplishment of the theory of relativity was that it brought the obvious principle of the relativity of motion into harmony with the existence of inertial forces. The Galilean law of inertia shows that there is a kind of obligatory guidance in the universe, which constrains a body left to itself to move with a perfectly definite motion, once it has been set in motion in a particular direction in the world. The body does this in virtue of a tendency of persistence, which carries on this direction at each instant "parallel to itself." At every position $\mathrm{P}$ in the universe, this tendency of persistence (the "guiding field") thus determines the infinitesimal parallel displacement of vectors from $P$ to world-points indefinitely near to $\mathrm{P}$. Such a continuum, in which this idea of infinitesimal parallel displacement is determinate, I have designated as an "affinely connected" one (affin zusammenhängend). According to the ideas of Galileo and Newton, the "affine connection" of the universe (the difference between straight and curved) is given by its geometrical structure. A vector at any position in the universe determines directly and without ambiguity, at every other position, and by 